\title{
La observación del sexismo en el patio escolar del CEIP Víctor Oroval y Tomás: un estudio de casos
}

\author{
The observation of sexism in the playground at Victor Oroval \\ primary school: a case study
}

Sandra Molines Borrás

Recibido: 8/01/2019

Aceptado: 22/12/2020

\section{RESUMEN}

El estudio empírico que se presenta contribuye a la detección del sexismo en la escuela a través de la observación sistemática de los comportamientos del alumnado en el patio de recreo. Los principales objetivos consisten en detectar las diferencias en el uso del espacio, observar la distinta tipología de juegos desempeñadas por el alumnado de distinto sexo y analizar la disposición de materiales de juego facilitadas por la escuela (CEIP Víctor Oroval i Tomás) ubicada en Carcaixent, Valencia. La metodología utilizada ha consistido en un estudio descriptivo de casos, en el que han participado un total de 142 alumnos/as. Los resultados obtenidos permiten extraer diferencias en todos los campos observados. Se plantean como propuestas necesarias para la investigación futura introducir diagnósticos con perspectiva de género sobre el uso del patio de recreo, por un lado, y, por otro, la incorporación de medidas concretas de corrección para evitar el sexismo escolar.

Palabras clave: escuela coeducativa, igualdad de oportunidades, patio de recreo, sexismo, género.

\section{ABSTRACT}

The empirical study that is presented tries to contribute to the detection of the sexism in the school through the systematic observation of the children's behaviors in the playground. Specifically, the main objectives are to detect differences in the use of space, to observe the different types of games played by students of different sexes and to analyze the disposition of play materials provided by the school (CEIP Víctor Oroval i Tomás) located in Carcaixent, Valencia. The methodology used has consisted of a descriptive case study, in which a total of 142 students participated. The results obtained allow to extract differences in each and every one of the fields observed. The impact of gender stereotypes, as well as self-perception and self-efficacy in the game, determine a situation of inequality among students. Proposals for future research are suggested both to introduce diagnoses with a gender perspective on the use of the playground, and to incorporate concrete measures of correction to avoid school sexism.

Keywords: coeducation school; equal opportunity; playground; schoolyard; sexism and gender. 


\section{INTRODUCCIÓN}

Observar el sexismo escolar se ha ido convirtiendo en una tarea difícil que pocos centros educativos sienten la necesidad de abordar. Varias son las razones que pueden justificar esta situación, por un lado la escasa formación en coeducación o perspectiva de género así como en metodología de investigación que suele tener el profesorado (Anguita y Torrego, 2009; Moreno, 2013 y Mayorga et al. 2014). Por otro, la creencia compartida de que no son sexistas y que tal y como se especifica en sus Proyectos Educativos de centro, en sus escuelas se trabaja la igualdad de oportunidades y se evita y elimina cualquier tipo de discriminación (Santos, 2010).

Sin embargo, como cualquier otro entorno social, las escuelas están íntimamente afectadas y condicionadas por la cultura en la que están inscritas así como por el discurso dominante que las direcciona, Connell (2001, citado en Villar et al. 2014). Tanto las infraestructuras de los centros (en especial la configuración de sus patios escolares), como el uniforme preestablecido, el lenguaje utilizado, los materiales curriculares seleccionados, etc, tienen un poderoso impacto no solo en los aprendizajes formales, sino en el currículum oculto que está asimilando el alumnado desde los primeros cursos de educación infantil (López, 2014) y todos ellos están íntimamente atravesados por el aprendizaje del género. La forma particular en la que el alumnado aprende los estereotipos y roles acordes a su cultura y sexo de nacimiento, afectan lo que este desea, piensa, actúa y siente.

Por todo ello hace falta que el profesorado adquiera una nueva identidad e incorpore nuevas funciones a las tradicionalmente ejercidas en el aula (Vega et al. 2013). Es imprescindible que el profesorado reflexione y desarrolle estrategias de cambio escolar que puedan llevarse a cabo en su contexto más cercano. Bonal (1997), a tal fin destaca que:

- A pesar de que el sexismo está presente también en el currículum explícito, principalmente se trata de un tipo de influencia y discriminación invisible.

- El sexismo es un problema del que normalmente no son conscientes ni el profesorado, ni el alumnado, ni las familias. 
- Las mujeres y las niñas ven limitadas, no formalmente pero sí realmente, sus preferencias, gustos, toma de decisiones, etc. La escuela, y con ella sus patios, solamente valoran una forma cultural concreta, la asociada al género masculino, e ignora o silencia la cultura femenina.

Para poder llevar a cabo actuaciones coeducativas, el profesorado necesita la supervisión de su nivel de conocimientos y su capacidad de toma de conciencia y análisis de la realidad con perspectiva de género (Aristizabal et al. 2018 y García et al. 2014). Si este colectivo no es capaz de diagnosticar la desigualdad, difícilmente será capaz de poder corregirla (Vega, 2012). Necesita también, “identificar los rasgos de género en su propio 'yo', y también qué, cuándo y cómo los transmite, para poder controlar esta transmisión" (Ferreiro, 2017, p.159).

El impacto de los estereotipos de género en los juegos infantiles ha sido altamente estudiado y demostrado en diferentes investigaciones como las aportadas por (Lobato, 2007; Cherney y London, 2006; Renold, 1997; Robinson y Morris, 1986; y Miller 1987) y sin embargo no parecen tener una repercusión tal que promuevan una reflexión interna en los centros, ni el profesorado parece tener conocimientos suficientes en estas materias para poderlos corregir (Rebollo et al. 2009). Tal y como afirma Tomé (2017) “en muchos casos, la falta de conocimiento y de respuesta hace que miren para otro lado, no saben ni por dónde empezar" (p.114).

\subsection{El patio y sus pistas deportivas: escenario ideal de observación}

El patio se configura habitualmente, como un espacio de pedagogía del género en donde se proclaman unas relaciones de poder desigual y de subordinación femenina que se van interiorizando y que trascienden la supuesta equidad formal de nuestro sistema educativo (Maceira, 2005; Rodríguez y García, 2009). Por otro lado, cabe tener en cuenta la importancia de la mayoría de las relaciones e interacciones que se configuran durante la práctica de deporte en los patios escolares, así como en las clases de educación física (Murillo et al. 2014), ya que éstas tienden a ser asimétricas y merecen una profunda reflexión (López, 2012; Cantó y Ruiz, 2005).

La homogeneidad de la masculinidad hegemónica resta valor a las diferencias y a las potencialidades individuales, puesto que no tiene en consideración las preferencias, los intereses y los talentos de otros grupos a los que no les gustan 
las actividades deportivas ni de competición (Sabuco et al. 2013). Por todo ello, tal y como proponen Subirats y Tomé (2010) el patio de juegos es un espacio especialmente interesante para investigar porque:

a) El profesorado no suele contar con estrategias de diagnóstico con perspectiva de género para estos espacios educativos y por ello suele percibir como normal, la distribución de los espacios y la dotación de las infraestructuras.

b) Desde la etapa infantil niñas y niños aprenden (por efecto de su diferente socialización), que la práctica deportiva es una actividad mayoritariamente masculina (Isorna et al. 2014; Moreno 2006; Rebollo y Martos, 1998).

En conclusión, si observamos nuestro entorno (barrio, ciudad, población, etc.) con mucha probabilidad nos encontraremos con un patio pensado para satisfacer las necesidades del alumno "tipo": niño con preferencias de juego activo y competitivo (Skelton, 2000), mientras que otro tipo de configuraciones más diversas de estos espacios serán difíciles de localizar.

\section{MÉTODO}

Dado que toda la información y sujetos de la investigación están circunscritos en un mismo espacio y comparten el mismo contexto educativo, este trabajo se engloba dentro de la metodología de estudio de casos. En este tipo de trabajos quien ostenta el rol de persona investigadora estudia una sola situación en profundidad (en este ejemplo el patio de una escuela) y fruto de este análisis puede dibujar un corpus de casos similares como base para la exploración y el desarrollo de una teoría (Edwards, 1998). Dicha metodología es idónea para el estudio de las ciencias sociales y en concreto es muy apropiada en investigaciones que tienen como propósito el análisis de un contexto educativo. Algunas de las ventajas de este tipo de investigaciones según Cohen y Manion (1990) y Martínez (1988) son:

- Representan la realidad tal y como sucede y por ello proporcionan una base natural para la generalización a partir del estudio de un ejemplo.

- Se representan verdades sociales dentro de microcosmos particulares.

Están centrados en los niveles "micro" de un sistema, por ejemplo una escuela y las interacciones particulares y concretas que se desarrollan en esos espacios entre los diferentes sujetos objeto de la investigación. 
Por todo ello este trabajo se enmarcaría como un estudio descriptivo. La investigación descriptiva se preocupa de cómo lo que es o lo que existe se relaciona con algún hecho precedente (en nuestro caso el sexismo), Best (1970 citado en Cohen y Manion 1990). Desde otro punto de vista también acorde a los principios de esta investigación se podría englobar el presente trabajo en la definición de una investigación etnográfica. Este tipo de investigaciones tratan de describir los sucesos que ocurren dentro de la vida de un grupo, con especial atención a las estructuras, jerarquías e interacciones sociales y a la conducta de los individuos respecto a su pertenencia al grupo y a la cultura dominante (Lukas y Santiago 2004), en este caso en el espacio del patio escolar.

Entre los campos de interés de este tipo de investigaciones etnográficas estarían por ejemplo, el cambio en las relaciones sociales y la formación que puede comportar tanto para el alumnado como para el profesorado, la toma de decisiones, la comunicación, la disposición de los medios y los recursos (Woods, 1987).

Por último, como estrategia de recogida de información se hace uso de la metodología observacional no participante. Este método consiste en el registro sistemático y la cuantificación del comportamiento tal y como se produce en situaciones naturales. Al suponerse no participante, se reduce el sesgo producido por los sujetos observados puesto que no existe ningún tipo de interacción voluntaria o involuntaria entre sujetos observados-persona observadora (Anguera, et., al 1993).

En este caso se confecciona una plantilla de trabajo u hoja de observación con un dibujo del patio con sus correspondientes zonas de juego. La plantilla utilizada en esta investigación se basó en las propuestas en el libro de Marina Subirats y Amparo Tomé (2010) titulado Balones fuera en el apartado de anexos: Instrumentos de observación del sexismo en el patio de juegos. Este tipo de técnica evita toda opción a reactividad puesto que el alumnado no es consciente de que su conducta está siendo registrada en ningún momento. Según Campbell (1986) esta investigación se puede definir, como aquella que lleva a cabo "un observador que anota una característica sorprendente de una cultura y luego dispone de todas las demás diferencias en todas las otras variables para tratar de hallar una explicación" (p. 87). 
En concreto la observación se llevó a cabo durante 15 días ininterrumpidamente tal y como se muestra en la exposición de resultados. Se tomó nota de los datos en un momento puntual de la hora del patio, en concreto a mitad del mismo. No se tuvo en cuenta el cambio en los juegos o en las agrupaciones, sino que se registró en un momento preciso lo que sucedía y era objeto de este trabajo.

\subsection{Objetivos}

Por todo ello, los principales objetivos de esta investigación han sido:

- Contabilizar la ocupación diferenciada del espacio del recreo escolar por parte de las niñas y de los niños.

- Analizar el tipo de juego que desarrollan en esos espacios clasificando los mismos en juegos activos o pasivos físicamente $y$ juegos estereotipadamente masculinos o femeninos.

- Conocer si el análisis de la propia configuración del espacio así como la dotación de recursos que ofrece el mismo puede favorecer una tipología de juegos y con ello la reproducción de un esquema y roles de género determinados (valoración de las infraestructuras).

\subsection{Población y muestra}

En este estudio ha participado todo el alumnado de primaria de la escuela objeto de análisis, tal y como se describe en la tabla 1.

Tabla 1. Alumnado matriculado en primaria. Molines Borrás, S. (2016). La Coeducación en un centro educativo: análisis del patio escolar. Tesis doctoral.

\begin{tabular}{rccc}
\hline Cursos & $\mathbf{N}^{\mathbf{0}}$ Alumnas & $\mathbf{N}^{\mathbf{0}}$ Alumnos & Total alumnado \\
\hline $\mathbf{1}^{\mathbf{0}}$ Primaria & 8 & 13 & 21 \\
$\mathbf{2}^{\mathbf{o}}$ Primaria & 13 & 7 & 20 \\
$\mathbf{3}^{\mathbf{o}}$ Primaria & 13 & 14 & 27 \\
$\mathbf{4}^{\mathbf{0}}$ Primaria & 11 & 9 & 20 \\
$\mathbf{5}^{\mathbf{0}}$ Primaria & 10 & 13 & 23 \\
$\mathbf{6}^{\mathbf{0}}$ Primaria. & 15 & 16 & 31 \\
Grupos A y B & & & \\
\hline & 70 & 72 & 142 \\
\hline
\end{tabular}

Este centro educativo se caracteriza por la multiculturalidad en la configuración de la comunidad educativa. Esta característica puede relacionarse con las faltas de asistencia por parte del alumnado cuando festejan algunos días 
conmemorativos propios de sus países de nacimiento y por ello no acuden al centro.

\section{DESCRIPCIÓN DEL C.E.I.P. VICTOR OROVAL Y TOMÁS Y DE SU PATIO DE RECREO}

El CEIP Víctor Oroval y Tomás, es un centro de titularidad pública y de una única línea ubicado en la población de Carcaixent (Valencia). Entre las señas de identidad de su proyecto educativo tal y como se puede extraer de su página web destaca que:

- $\quad$ Se reconoce como un centro que trata de atacar el sexismo y de promover la igualdad de oportunidades en todos los niveles y esferas educativas.

Cabe decir en este punto que en el momento de la realización de la investigación, no existía ninguna intervención conducente a disminuir el impacto del sexismo en el patio escolar.

El patio se estructura en cinco zonas bien diferenciadas. En la tabla 2 se muestran las características de cada una de ellas.

Tabla 2.

Zonas del patio escolar. Molines Borrás, S. (2016). La Coeducación en un centro educativo: análisis del patio escolar. Tesis doctoral

\begin{tabular}{|c|c|}
\hline ZONA & CARACTERÍSTICAS \\
\hline ZONA 1 & \\
\hline $\begin{array}{l}\text { Dimensiones: } \\
1062 \mathrm{~m}^{2} \text { : } \\
51.9 \%\end{array}$ & $\begin{array}{l}\text { Esta es la zona donde se sitúa el campo de } \\
\text { fútbol. Viene determinada por las rayas y el } \\
\text { dibujo que delimita las extensiones de un } \\
\text { campo de fútbol así como por las dos } \\
\text { porterías. Es la zona más extensa. }\end{array}$ \\
\hline
\end{tabular}

ZONA 2

Dimensiones: $685,2 \mathrm{~m} \div 33.5 \%$

Es la zona de la pista de baloncesto. Tiene las rayas en el suelo que delimitan este tipo de deporte así como dos canastas que de forma estable están situadas siempre en el mismo lugar. Puesto que el campo de baloncesto es más pequeño que el del fútbol en el espacio sobrante está dibujado un campo para el juego del "cementerio".

ZONA 3

Dimensiones:

$98.23 \mathrm{~m}^{2}$ :

$4,8 \%$
Esta zona se caracteriza por ser pequeña en extensión, periférica, con suelo de tierra y
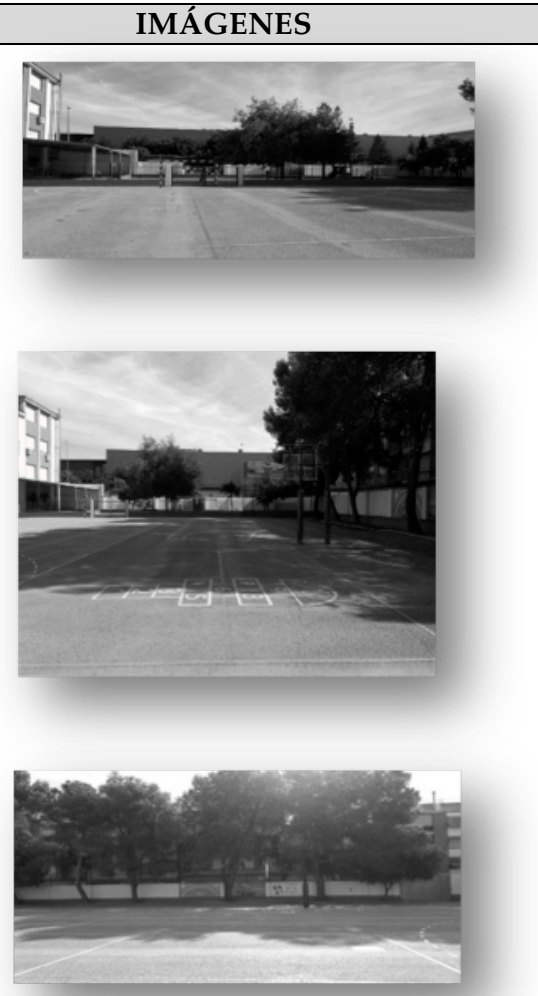
arbolada (por lo que proporciona sombra en días de sol).

Al igual que las zonas 4 y 5, es una zona periférica.

ZONA 4

Dimensiones:

200,94 m:9.8\%

Esta zona presenta como principal característica que tiene un porche sustentado por unas columnas y que por ello protege del sol. También es la única zona que presenta 4 bancos para sentarse.

ZONA 5

Esta zona se define como el muro y la valla que separa la zona de infantil de la de primaria, no se supone un espacio de juego en sí mismo pero allí se ubica parte del alumnado diariamente y por eso se ha considerado conveniente incluirla en el registro de observación.
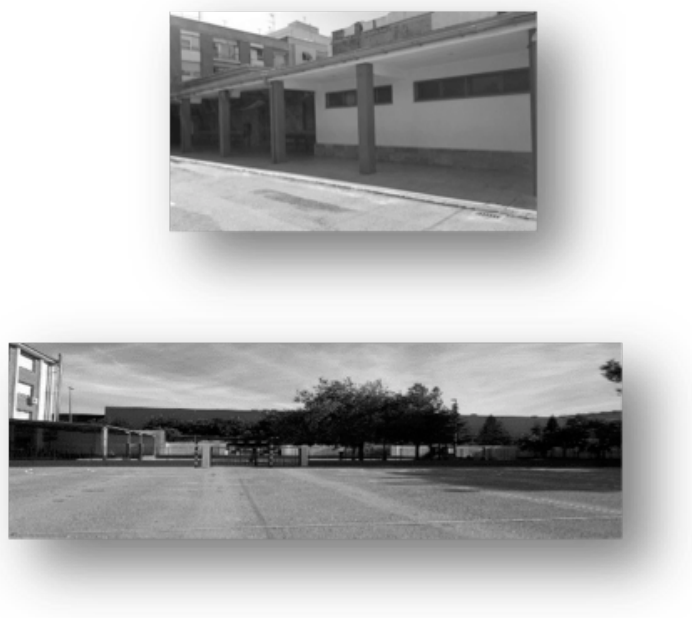

Como resumen de la tabla anterior, las zonas de mayor extensión ( 1 y 2 ) son las zonas centrales ocupando un $85,4 \%$ del patio y destinadas a pistas deportivas, mientras que las zonas de menor extensión (3, 4 y 5) con el restante 14,6\% del espacio son las zonas periféricas. Estas no están habilitadas con ningún tipo de infraestructura particular salvo los bancos para sentarse.

\section{RESULTADOS}

La información acerca de las conductas consideradas más habituales en el patio del colegio por parte de niñas y niños pretende dar respuesta a los tres objetivos fijados con anterioridad en este trabajo. La cuantificación de toda esta información queda recogida en las tablas 3, 4, 5, 6, 7, 8 y 9.

Tabla 3. Número de alumnado observado en el patio por día y sexo. Molines Borrás, S. (2016). La Coeducación en un centro educativo: análisis del patio

\begin{tabular}{|l|l|l|l|l|l|l|l|l|l|l|l|l|l|l|l|l|l|}
\hline Día & $\mathbf{1}$ & $\mathbf{2}$ & $\mathbf{3}$ & $\mathbf{4}$ & $\mathbf{5}$ & $\mathbf{6}$ & $\mathbf{7}$ & $\mathbf{8}$ & $\mathbf{9}$ & $\mathbf{1 0}$ & $\mathbf{1 1}$ & $\mathbf{1 2}$ & $\mathbf{1 3}$ & $\mathbf{1 4}$ & $\mathbf{1 5}$ & Media & $\begin{array}{l}\text { Desv. } \\
\text { Estándar }\end{array}$ \\
\hline Niños & 61 & 46 & 51 & 54 & 59 & 62 & 48 & 44 & 44 & 38 & 61 & 59 & 54 & 56 & 67 & 53.6 & 8.2 \\
\hline Niñas & 56 & 56 & 53 & 54 & 58 & 50 & 62 & 44 & 43 & 45 & 49 & 47 & 56 & 49 & 50 & 51.5 & 5.5 \\
\hline Total & 117 & 102 & 104 & 108 & 117 & 112 & 110 & 88 & 87 & 83 & 110 & 106 & 111 & 105 & 117 & 105.1 & 13.7 \\
\hline
\end{tabular}

Niños: $n^{\circ}$ de niños observados en el patio Niñas: $n^{\circ}$ de niñas observadas en el patio 
Los días 8, 9 y 10 de observación, la clase de $3^{\circ}$ curso de primaria estuvo castigada sin patio por mal comportamiento, de ahí que el número de alumnado registrado sea significativamente menor. Este dato afecta a la media total y aumenta el valor de la desviación típica.

El primer objetivo centra su atención en la observación sistemática de la ocupación diferencial del espacio de recreo, asumiéndose como hipótesis de partida la obtención de diferencias claras entre niñas y niños. Los resultados obtenidos a través del procedimiento de la observación no participante confirman la hipótesis inicial.

Tabla 4. Media y desviación típica de alumnado por zona y sexo. Molines Borrás, S. (2016). La Coeducación en un centro educativo: análisis del patio escolar. Tesis

\begin{tabular}{|c|c|c|c|c|}
\hline & Media Niños & $\begin{array}{l}\text { Desviación } \\
\text { Típica Niños }\end{array}$ & Media Niñas & $\begin{array}{l}\text { Desviación } \\
\text { Típica Niñas }\end{array}$ \\
\hline Zona 1 & 21.73 & 3.71 & 5.67 & 1.76 \\
\hline Zona 2 & 19.40 & 6.47 & 17.53 & 5.51 \\
\hline Zona 3 & 4 & 4.46 & 8.40 & 4.94 \\
\hline Zona 4 & 5.53 & 2.53 & 17 & 5.37 \\
\hline Zona 5 & 2.93 & 2.81 & 2.93 & 2.69 \\
\hline
\end{tabular}

Tabla 5. Media de alumnado por zona central o periférica y sexo. Molines Borrás, S. (2016). La Coeducación en un centro educativo: análisis del patio escolar. Tesis

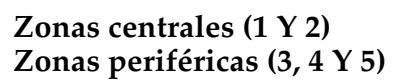

\begin{tabular}{ll}
\hline Media Niños & Media Niñas \\
\hline 41.13 & 23.20 \\
12.46 & 28.33
\end{tabular}

Tal y como se observa en los datos numéricos recogidos en las tablas 4 y 5, en la zona 1 del patio, la más grande, hay casi cuatro veces más presencia de niños que de niñas. Es la zona reservada para el fútbol y no comparte su espacio con ningún otro juego, lo que muestra un gran privilegio de esta actividad frente a otras. En la zona 2 se observa una representación más o menos similar de alumnado de distinto sexo, aunque se observan grandes diferencias en cuanto a la tipología de juegos, como se comentará a continuación. Respecto de las zonas más pequeñas y periféricas o las zonas laterales (3 y 4) se observa una considerable mayor presencia de niñas que de niños, tratándose de zonas que por su configuración espacial predisponen antes al juego pasivo que al activo físicamente. No existen diferencias numéricas en la zona 5. 
Respecto al segundo objetivo, consistente en analizar el tipo de juegos (activo/pasivo, por un lado y masculino/femenino, por otro) que se desarrollan durante el recreo, la hipótesis de partida asumía diferencias significativas, con un predominio de juegos activos y estereotipadamente masculinos por parte de los niños mientras que en las niñas iban a ser dominantes las actividades lúdicas pasivas y estereotipadamente femeninas. Para delimitar la actividad o pasividad en el juego, cabe tener en cuenta la definición de actividad física utilizada por Devis (2000 citado en Frago, 2015) quien la define como cualquier movimiento corporal intencional, realizado con los músculos esqueléticos que requiere y produce un gasto de energía y que propicia una experiencia personal permitiéndonos interactuar con los seres y el ambiente que nos rodea. A continuación se muestran los resultados al respecto:

Tabla 6. Media y desviación típica de alumnado con juego activo o pasivo físicamente y sexo. Molines Borrás, S. (2016). La Coeducación en un centro educativo: análisis del patio escolar. Tesis doctoral

\begin{tabular}{lllllllll}
\cline { 2 - 10 } & $\begin{array}{c}\text { Media } \\
\text { Niños } \\
\text { juego } \\
\text { activo }\end{array}$ & $\begin{array}{c}\text { Desv. } \\
\text { Típica } \\
\text { Niños } \\
\text { juego } \\
\text { activo }\end{array}$ & $\begin{array}{c}\text { Media } \\
\text { Niñas } \\
\text { juego } \\
\text { activo }\end{array}$ & $\begin{array}{c}\text { Desv. } \\
\text { Típica } \\
\text { Niñas } \\
\text { juego } \\
\text { activo }\end{array}$ & $\begin{array}{l}\text { Media } \\
\text { Niños } \\
\text { juego } \\
\text { pasivo }\end{array}$ & $\begin{array}{c}\text { Desv. } \\
\text { Típica } \\
\text { Niños } \\
\text { juego } \\
\text { pasivo }\end{array}$ & $\begin{array}{c}\text { Media } \\
\text { Niñas } \\
\text { juego } \\
\text { pasivo }\end{array}$ & $\begin{array}{c}\text { Desv. } \\
\text { Típica } \\
\text { Niñas } \\
\text { juego } \\
\text { pasivo }\end{array}$ \\
\hline Zona 1 & 21.13 & 3.46 & 4.73 & 2.15 & 0.60 & 1.30 & 0.93 & 1.83 \\
Zona 2 & 18.33 & 6.72 & 11.47 & 6.12 & 1.07 & 1.94 & 6.07 & 4.46 \\
Zona 3 & 0.33 & 1.29 & 0.47 & 1.36 & 3.67 & 4.48 & 7.93 & 4.65 \\
Zona 4 & 0.67 & 1.50 & 3.47 & 4.29 & 4.87 & 2.90 & 13.53 & 6.64 \\
Zona 5 & 0 & 0 & 0 & 0 & 2.87 & 2.88 & 3.14 & 2.66 \\
Total & 40.46 & 12.97 & 20.14 & 13.92 & 13.08 & 13.5 & 31.6 & 20.24
\end{tabular}

El criterio de clasificación como juego estereotipadamente masculino o

femenino es aquel en el que la proporción de niñas o niños está fuera del rango (40\%-60\%). Por debajo de un 40\% de presencia de un determinado sexo se considera que ese juego está especialmente segregado por alguna razón. En este caso los estereotipos constituyen herramientas socioculturales sobre las que se asientan normas de funcionamiento social, como las que determinan los juegos, a la vez que sirven de referente para estructurar la identidad de los sujetos (Colás y Villaciervos, 2007). Por todo ello, dependiendo de si eres chico o chica, sería aceptado como "normal y deseable" que jugases a uno u otro tipo de juego (Zaro, 1999). Los resultados obtenidos, expuestos en las tablas 7, 8 y 9 confirman nuestra hipótesis sobre la presencia de los estereotipos en los datos de la observación. 
Tabla 7. Juegos estereotipadamente masculinos. Molines Borrás, S. (2016). La Coeducación en un centro educativo: análisis del patio escolar. Tesis doctoral

\begin{tabular}{|c|c|c|c|c|c|c|c|}
\hline & Media & $\%$ & & $\mathbf{M e}$ & $\%$ & \multicolumn{2}{|c|}{ Media } \\
\hline $\begin{array}{l}\text { Futbol } \\
\text { chicos }\end{array}$ & 21 & 82.35 & $\begin{array}{l}\text { Básquet } \\
\text { chicos }\end{array}$ & 6 & 76.92 & $\begin{array}{l}\text { Perseguir } 8.9 \\
\text { pelota } \\
\text { chicos }\end{array}$ & 68.46 \\
\hline $\begin{array}{l}\text { Futbol } \\
\text { chicas }\end{array}$ & 4.5 & 17.65 & $\begin{array}{l}\text { Básquet } \\
\text { chicas }\end{array}$ & 1.8 & 23.08 & $\begin{array}{l}\text { Perseguir } 4.1 \\
\text { pelota } \\
\text { chicas }\end{array}$ & 31.54 \\
\hline
\end{tabular}

Tabla 8. Juegos estereotipadamente femeninos. Molines Borrás, S. (2016). La Coeducación en un centro educativo: análisis del patio escolar. Tesis doctoral

\begin{tabular}{|c|c|c|c|c|c|c|c|c|c|c|c|}
\hline & Media & $\%$ & & Media & $\%$ & & Media & $\%$ & & Media & $\%$ \\
\hline $\begin{array}{l}\text { Cuerda } \\
\text { chicos }\end{array}$ & 1.67 & 15.66 & $\begin{array}{l}\text { Baile } \\
\text { chicos }\end{array}$ & 0 & 0 & $\begin{array}{l}\text { Torito } \\
\text { en alto } \\
\text { chicos }\end{array}$ & 2.5 & 35.72 & $\begin{array}{l}\text { Cementerio } \\
\text { chicos }\end{array}$ & 3 & 36.15 \\
\hline $\begin{array}{l}\text { Cuerda } \\
\text { chicas }\end{array}$ & 9 & 84.34 & $\begin{array}{l}\text { Baile } \\
\text { chicas }\end{array}$ & 3.25 & 100 & $\begin{array}{l}\text { Torito } \\
\text { en alto } \\
\text { chicas }\end{array}$ & 4.5 & 64.28 & $\begin{array}{l}\text { Cementerio } \\
\text { chicas }\end{array}$ & 5.3 & 63.85 \\
\hline
\end{tabular}

Los números muestran con claridad una mayor prevalencia en las agrupaciones lúdicas por alumnado del mismo sexo. El porcentaje de juegos activos en los niños es muy superior al de las niñas. Junto a la clasificación de juegos recogida en las tablas 7 y 8 , se ha incluido en la observación otra serie de actividades que, si bien no se corresponden con ningún juego, sí engloban conductas bastante comunes entre el alumnado (Ver tabla 9). Se trata de los registros de las conductas de pasear, mirar el juego de otras personas, mirar el patio de infantil, conversar y estar en los bancos o en el suelo sentados/as. Este tipo de conductas se ha contabilizado dentro de la etiqueta de juegos pasivos en el registro general. El alumnado que se observa en estas conductas se suele ubicar en las zonas periféricas. Se obtienen los siguientes datos a este respecto:

Tabla 9. Otras actividades. Molines Borrás, S. (2016). La Coeducación en un centro educativo: análisis del patio escolar. Tesis doctoral

\begin{tabular}{lll} 
& Media & $\%$ \\
\cline { 2 - 3 } $\begin{array}{l}\text { Chicos paseo, } \\
\text { mirar, charlar o }\end{array}$ & 12.73 & 30.32 \\
$\begin{array}{l}\text { sentados } \\
\begin{array}{l}\text { Chicas paseo, } \\
\text { mirar, charlar o }\end{array}\end{array}$ & 29.26 & 69.68 \\
sentadas & &
\end{tabular}

Los datos también ratifican el predominio de actividades lúdicas estereotipadas por razón de género. La actividad más elegida por ellas es la de pasear, mirar o charlar sentadas y lo suelen hacer siempre sin interrumpir los juegos activos de 
ellos. Mientras tanto ellos suelen jugar a juegos estereotipadamente masculinos que hacen uso de una pelota y que por ello controlan los mayores espacios del patio.

El último objetivo consiste en establecer una valoración de las infraestructuras del centro referidas a la configuración del espacio y a la dotación de recursos. Tal y como se muestra, las pistas deportivas (zona 1 y 2 ) que suponen el 85,4\% del espacio total, son las que están dotadas de una infraestructura determinada para una tipología de juego concreta. Están habilitadas con unas porterías de fútbol y con unas canastas de baloncesto, así como se delimita pintado en el suelo el campo de ambos deportes. Fruto de este análisis podemos concluir que el colegio dota de los materiales necesarios de los juegos que habitualmente hacen uso los niños, mientras que no acondiciona especialmente ni dota de espacios ni infraestructuras cuidadas para las actividades que normalmente hacen ellas, que son aproximadamente el 50\% de alumnado del centro.

\section{DISCUSIÓN Y CONCLUSIONES}

Del análisis realizado se derivan dos conclusiones fundamentales:

1) El reconocimiento de que la ocupación del espacio del recreo no es igualitaria para niñas y niños sino que resulta discriminatoria para las niñas. Conviene aclarar que con este estudio no se pretende negar la importancia que el desarrollo del deporte debe tener en la escuela. Al contrario, somos conscientes de la prioridad que las actividades físicas ejercen en el crecimiento holístico (físico y psicológico) de las personas, especialmente durante la niñez.

2) Evidenciar la existencia de una serie de actividades lúdicas, etiquetadas como estereotipadamente femeninas, que conviene poner en valor en la formación coeducativa porque son imprescindibles en el desarrollo evolutivo de niñas y niños.

Hemos naturalizado no solo la disposición e infraestructura con la que dotamos los patios, sino el uso diferenciado que ellas y ellos hacen de las mismas. Nos resulta normal y habitual observar patios androcéntricos que responden a los gustos y preferencias de cierta parte de alumnado (chicos futboleros y que juegan a pelota). Sin embargo, no hemos analizado en profundidad las posibles consecuencias que este hecho puede acarrear: 
- Ellas y ellos están desarrollando diariamente diferentes competencias en su tiempo libre de juego.

- El patio escolar genera y reproduce una jerarquía, orden y control por parte del colectivo que hace uso de ciertos espacios sobre otros. El poder y la sumisión se aprenden y reproducen diariamente en estos espacios.

- No se está educando hacia la convivencia y la diversidad, las agrupaciones en el juego suelen estar segregadas por sexo (Gil et al. 2017).

- La disposición de ciertas infraestructuras (porterías y canastas), reduce opciones en la tipología de juegos porque infravalora e impide la posibilidad de otras actividades.

La construcción de los estereotipos de género es una de las variables con mayor peso en la justificación de las diferencias observadas en esta investigación. Por un lado estos no solo están actuando como descriptores de la conducta, sino que presentan un elemento fuerza para prescribir lo que se considera correcto en ellos y ellas en su experiencia en el juego (Lameiras et al. 2004; Barberá, 2006) o, en otros, en su rendimiento (Bian et al. 2017). Los estudios llevados a cabo en las escuelas por Eliasson (2011) respecto del fútbol, la masculinidad y la preferencia de juego en los niños, vienen a corroborar esa idea. Sus investigaciones confirman que el poder del estereotipo del fútbol como juego masculino es tan fuerte, que incluso las chicas que juegan al fútbol no suelen considerarse ni se las considera igual de buenas que a ellos. En la misma línea se encuentran las investigaciones realizadas por Clark y Paechter (2007). El arquetipo viril al que están sometidos los niños comporta entre otros tipos de actividades y formas de mostrarse en el mundo, el hecho de ser bueno jugando al futbol (Lomas, 2007; Sanfélix, J. y Téllez, A. (2017), mientras que la construcción de la feminidad en ellas comporta otro tipo de deseos, actitudes y actividades muy distantes a este tipo de deporte y más vinculadas a la pasividad y al juego tranquilo (Puerta y González, 2013).

Por otro lado, diversas investigaciones muestran la existencia de diferencias entre alumnado de distinto sexo en dos variables: nivel de satisfacción con la realización de actividad física (a ellos les gusta más) y autopercepción respecto de su nivel de efectividad y eficiencia desarrollando actividades físicas (ellos se perciben mejores) y estas variables pueden estar impactando de un modo muy 
sutil e invisible en las diferentes preferencias mostradas por ambos sexos (Vallejo y González, 2012; Sánchez y Gómez, 2014)

Las personas construyen los espacios y los espacios construyen a las personas. Vivimos y trabajamos en las escuelas sin percatarnos de la influencia que los espacios tienen en los miembros de la comunidad escolar. El espacio está lleno de significados (López, 2018). Su configuración, su uso, su apertura/clausura, su ornamentación, constituyen una sintaxis en el discurso de la escuela. Dotamos de grandes superficies a determinadas actividades, juegos o deportes y dejamos de lado y con ello infravaloramos otro tipo de acciones más relacionadas con la conversación, el sosiego, el paseo o el juego pasivo físicamente (Saldaña, Goula y Cardona, 2020).

Para reducir el impacto de la apropiación del espacio por parte de los niños así como de las pelotas, y con ello mejorar la convivencia del patio, Martínez (2009) así como el colectivo Punt6 i Coeducacció (2020) aportan una serie de recomendaciones que persiguen en definitiva desmontar los abusos de poder inconscientes que imperan y determinan la situación en el recreo escolar. En dicho reordenamiento, sería de vital importancia la implicación de toda la comunidad educativa así como la toma de conciencia de la situación actual.

Del mismo modo, cabe atender a los resultados de las investigaciones llevadas a cabo por Yuen y Shaw (2003) cuando relacionan la vivencia en el juego y los usos de libertad en la infancia. En este caso ellas hablan de la importancia en el estudio de las repercusiones de dos tipologías de juegos: los juegos estructurados y los juegos no estructurados. Los juegos estructurados estarían más sujetos a las normas del juego creadas por adultos, serían juegos que las niñas y los niños llevan diariamente en sus mochilas de género puesto que son asimilados con el sexismo imperante en la sociedad y por lo tanto más fácilmente portadores de dicha carga. Se podrían incluir en este tipo de juegos al fútbol o el básquet. Se trata de actividades que el alumnado aprende desde el prisma de las personas adultas, puesto que ellas son las que mayormente las practican y las que actúan como modelos de referencia. Por otro lado están los juegos no estructurados, englobarían aquellos juegos creados e inventados por el propio alumnado y por este motivo sujetos en menor medida al impacto de la cultura adulta y a los estereotipos de género. Dichas autoras prevén que la estimulación de los juegos no estructurados puede promover una mayor 
igualdad en la jerarquía y disposición de los mismos, puesto que en su origen, poseen un mandato del sexismo social imperante de menor impacto que el resto de juegos socialmente inscritos en el modelo androcentrista y patriarcal.

Deconstruir los estereotipos de género es imprescindible si queremos diseñar nuevas socializaciones igualitarias que quiebren la idea del género como destino, la posibilidad de nuevos modelos de feminidad y masculinidad pasa, indudablemente, por cuestionar los mandatos culturales hegemónicos (Ruiz, 2017, p.172).

Cabe por ello reivindicar un espacio plural de igualdad de oportunidades y de solidaridad para el desarrollo de cualquier actividad y esto depende, en mayor medida, de los valores del centro, antes que de otro tipo de variables (Trachana, 2013). Si educar implica insertar un elemento crítico y ético a los procesos naturales de socialización (Santos, 2010), admitamos que en la mayoría de los patios no se está educando y que se está aprendiendo e interiorizando una serie de competencias y discursos que no favorecen el deseado camino hacia la igualdad de oportunidades.

Garantizar una formación transversal en género por parte del profesorado, se presupone una de las principales herramientas a desarrollar en los centros si se quiere superar a la escuela mixta para conseguir una verdadera escuela coeducativa (Vizcarra et al. 2015 y García et al. 2013). El saber escolar sigue transmitiendo, básicamente, una cultura androcéntrica, que no se ha transformado suficientemente para dar cabida a las aportaciones, los valores, las preferencias y las prácticas culturales tradicionalmente femeninas (Subirats, 2010). Tal vez, si miramos con perspectiva de género lo que está pasando en nuestros patios escolares, nos damos cuenta de la necesidad de educar en los mismos y de favorecer que estos espacios sean representativos de la inclusión y la diversidad de opciones, más que de la homogeneización de los gustos y de las preferencias de cierta parte del alumnado. 


\section{BIBLIOGRAFÍA}

Anguera, M.T., Behar, J., Blanco, A., Carreras, M.V., Losada, J.L., Quera, V. y Riba, C. (1993). Metodología observacional en la investigación psicológica, 2 Fundamentación. Barcelona: PPU, S.A.

Anguita, R. y Torrego, L. (2009). Género, educación y formación del profesorado. Retos y posibilidades. Revista Interuniversitaria de formación del profesorado, 23 (1), 17-25. Recuperado de: http: / / www.redalyc.org / articulo.oa?id=27418821002

Aristizabal, P., Gómez-Pintado, A., Ugalde, A. I. y Lasarte, G. (2018). La mirada coeducativa en la formación del profesorado. Revista Complutense de Educación, 29 (1), 79-95. doi: https: / / doi.org/10.5209/RCED.52031

Barberá E. (2006). Aportaciones de la psicología al estudio de las relaciones de género. En C. Rodríguez (Comp.), Género y currículo (pp. 59-76). Madrid: Ediciones Akal.

Bian, L., Leslie, S. y Cimpian, A. (2017). Gender stereotypes about intellectual ability emerge early and influence children's interests. Science, 355, 389391. doi: : https:// doi.org/10.1126/science.aah6524

Bonal, X. (1997). Las actitudes del profesorado ante la coeducación. Propuestas de intervención. Barcelona: Editorial Graó.

Campbell, D. (1986). Grados de libertad y el estudio de casos. En T.D. Cook y Ch. S. Reichardt, Métodos cualitativos y cuantitativos en investigación evaluativa (pp. 80-104). Madrid: Ediciones Morata.

Cantó, R. y Ruiz, L.M. (2005). Comportamiento motor espontáneo en el patio de recreo escolar: Análisis de las diferencias por género en la ocupación del espacio durante el recreo escolar. Revista Internacional de Ciencias del Deporte, 1, 28-45. https:/ / doi.org/10.5232/ ricyde2005.00103

Cherney, I. D. y London, K. (2006). Gender-linked differences in the toys, television shows, computer games, and outdoor activities of 5- to 13year-old. Sex roles, 54 (9), 717-726. doi: https:/ / doi.org/10.1007/s11199$\underline{006-9037-8}$ 
Clark, S. y Paechter, C. (2007). Why can't girls play football? Gender dynamics and the playground. Sport, education and society, 12 (3), 261-276. Doi: https: / / doi.org/10.1080/13573320701464085

Cohen, L. y Manion, L. (1990). Métodos de investigación educativa (3 $\left.{ }^{\mathrm{a}} \mathrm{Ed}\right)$. Madrid: La Muralla.

Colás, P. y Villaciervos, P. (2007). La interiorización de los estereotipos de género en jóvenes y adolescentes. Revista de Investigación Educativa, 25 (1), 35-38.

Col-lectiu Punt6 i Coeducacció (2020). Patios coeducativos: Guía para la transformación feminista de los espacios educativos. Recuperado de: https: / / issuu.com/patioscoeducativos/docs/libropatioscoeducativos_09 -04-2020_cast

Edwards, D. J. A. (1998). Types of case study work: A conceptual framework for case-based research. Journal of Humanistic Psychology, 38 (3), 36-70. doi: https: / / doi.org/10.1177/00221678980383003

Eliasson I. (2011). Gendered socialization among girls and boys in children's football teams in Sweden. Soccer $\mathcal{E}$ Society, 12 (6), 820-833. Doi: https: / / doi.org/10.1080/14660970.2011.609682

Ferreiro, L. (2017). (Co)Educación afectivo-emocional y sexual, para despatriarcalizar la escuela y caminar hacia la igualdad. Atlánticas. Revista Internacional de Estudios Feministas, 2 (1), 134-165. doi: https: / / doi.org/10.17979/arief.2017.2.1.1771

Frago, J. M. (2015). Niveles de actividad física en escolares de educación primaria: actividad física habitual, clases de educación física y recreos (Tesis Doctoral). Universidad de Zaragoza, Zaragoza, España.

García, R., Sala, A., Rodríguez, E. y Sabuco, A. (2013). Formación inicial del profesorado sobre género y educación: Impactos metacognitivos de la inclusión curricular transversal sobre sexismo y homofobia. Profesorado. Revista de currículum y formación del profesorado, 17, 269-287.

García-Pérez, R., Rebollo, M.A., Vega, L., Barragán-Sánchez, R.; Buzón, O. y Piedra, J. (2014). El patriarcado no es transparente: competencias del profesorado para reconocer desigualdad. Cultura y Educación 2 (3), 385397. Doi: https: / / doi.org/10.1174/113564011797330298 
Gil, P., Valdivia, P., González, S. y Zagalaz, M. L. (2017). Percepciones y comportamientos de discriminación sexual en la práctica de ejercicio físico entre los hombres y las mujeres preadolescentes en el tiempo de ocio. Revista de Psicología del Deporte, 26 (2), 81-86.

Isorna, M., Rial, A, y Vaquero, R. (2014). Motivaciones para la práctica deportiva en escolares federados y no federados. Retos. Nuevas tendencias en Educación Física, Deporte y Recreación, (25), 80-84.

Lameiras, M., Rodríguez, Y., Ojea, M. y Dopereiro, M. (2004). Fundamentación teórica. En M. Lameiras (Coord.), Programa coeducativo de desarrollo psicoafectivo y sexual. (pp. 29-36). Madrid: Pirámide.

Lobato, E. (2007). Juego y género. Estudio etnográfico sobre la construcción de la identidad del género en contextos y prácticas lúdicas infantil (Tesis doctoral). Universidad de Oviedo, España.

Lomas, C. (2007). ¿La escuela es un infierno? Violencia escolar y construcción cultural de la masculinidad. Revista de educación, 342, 83-101.

López, R. (2012). La coeducación en el área de educación física: revisión, análisis y factores condicionantes. Lecturas: Educación Física y Deportes, Revista Digital (169). Recuperado de: http:/ / www.efdeportes.com/efd169/lacoeducacion-en-educacion-fisica.htm.

López, A. (2014). Análisis de la ausencia de las mujeres en los manuales de la ESO: una genealogía de conocimientos ocultada. Revista de Educación, 363, 282-308. https: / / doi.org/10.4438/1988-592X-RE-2012-363-188

López, S. (2018). Esencia. Diseño de espacios educativos. Zaragoza: Edelvives.

Lukas, J. F. y Santiago, K. (2004). Diseños de investigación aplicados a la evaluación. En J.F. Lukas y K. Santiago. Evaluación educativa (pp.177206). Madrid: Alianza Editorial.

Maceira, L. (2005). Investigación del currículo oculto en la educación superior: alternativa para superar el sexismo en la escuela. Revista de estudios de género. La ventana, 21, 187-227. Recuperado de: http:/ / www.redalyc.org/ articulo.oa?id=88402107

Martínez, J. (1988). El estudio de casos en la investigación educativa. Investigación en la escuela, 6, 41-50. Facultad de Filosofía y C.C. de la educación.

Recuperado

de:

http: / / www.investigacionenlaescuela.es / articulos /6/R6_3.pdf 
Martínez, C. (2009). Algunas propuestas para trabajar la convivencia en primaria. En S. Funes (Coord.) Gestión eficaz de la convivencia en los centros educativos (pp. 121-163). Madrid: Wolters Kluwer España.

Mayorga, Ma. J., Santos, M. A. y Madrid, D. (2014). Formación y actualización de la función docente. Diálogos pedagógicos, 24 (12), 11-28.

Moreno, J. A. (2006). Actitudes hacia la práctica físico-deportiva según el sexo del practicante. International Journal of Sport Science, 3, 20-43. doi: https: / / doi.org/10.5232/ ricyde2006.00302

Moreno, M. A. (2013). Queremos coeducar. Materiales de apoyo a la acción coeducativa. Consejería de Educación, Cultura y Deporte. Dirección General de Políticas Educativas y Ordenación Académica. Centro de profesorado y recursos de Avilés-Occidente. Recuperado de: http: / / blog.educastur.es/marianmoreno/files/2013/06/ queremoscoe ducar-2.pdf.

Miller, C. L. (1987). Qualitative differences among gender-stereotyped toys: Implications for cognitive and social development in girls and boys. Sex roles, 16 (9), 473-487. doi: https: / / doi.org/10.1007/BF00292482

Murillo, B., Julián, J.A., García-González, L., Albarca-Sos, A. y Zaragoza, J. (2014). Influencia del género y de los contenidos sobre la actividad física y la percepción de competencia en Educación Física. RICYDE. Revista internacional de ciencias del deporte, 36 (10), 131-143. doi: http:/ / dx.doi.org/10.5232/ ricyde2014.03604

Puerta, S. y González, E. M. (2013). Reproducción de los estereotipos de género en Educación Infantil a través de los juegos y juguetes. Investigación en la escuela, 85, 63-74. doi: https: / / doi.org/10.12795/IE.2015.i85.05

Rebollo, M. Á., García, R., Ruiz, E., y Pérez, C. (2009). Tecnologías para la coeducación y la igualdad: el Proyecto Teon XXI. En I. Vázquez (Coord.). Conferencia llevada a cabo en el Congreso Universitario Andaluz Investigación y Género. Sevilla.

Rebollo, S. y Martos, P. (1998). Práctica físico-deportiva y género. Aproximación a una realidad marginal. Revista Motricidad, 4, 183-202.

Renold, E. (1997). All they've got on their brains is football. Sport, masculinity and the gendered practices of playground relations. Sport, education and society, 2(1), 5-23. doi: https:/ / doi.org/10.1080/1357332970020101 
Robinson, C. C. y Morris, J. T. (1986). The gender-stereotyped nature of Christmas toys received by 36-48, and 60-month-old children: A comparison between nonrequested vs requested toys. Sex roles, 15(1), 21-32. doi: https: / / doi.org/10.1007/BF00287529

Rodríguez, H. y García, H. (2009). Asimilación de códigos de género en las actividades del recreo escolar. Revista Interuniversitaria de formación del profesorado, $64(23,1) 59-72$.

Ruíz Repullo, C. (2017). Estrategias para educar en y para la igualdad: coeducar en los centros. Atlánticas. Revista Internacional de Estudios Feministas, 2 (1), 166-191. doi: https: / / doi.org/10.17979/arief.2017.2.1.2063

Sabuco, A., Sala, A., Santana, R. y Rebollo, Ma . A. (2013). Discursos de niños varones sobre la masculinidad en contextos escolares. Un estudio Piloto. Profesorado, 17 (1), 141-157.

Saldaña, D., Goula, J. y Cardona, H. (2020). La coeducació com a pràctica espacial. Guix. Elements d'Acció Educativa, 10-14, 468-469.

Sánchez, B. J. y Gómez A. (2014). Autoconcepto físico en una muestra de estudiantes de primaria y su relación con el género y la práctica deportiva extraescolar. E-balonmano.com. Revista de Ciencias del Deporte, 10(2), 113-120. Recuperado de: http: / / www.redalyc.org/ articulo.oa?id=86532543005.

Sanfélix, J. y Téllez, A. (2017). Lógicas prácticas en el proceso de construcción de la masculinidad de los hombres valencianos: callo, riesgo, fútbol y arca. MCS Masculinities and Social Change, 6, (2), 96-118. Doi: https: / / doi.org/10.17583/mcs.2017.1937

Santos, M. A. (2010). Una pretensión problemática: educar para los valores y preparar para la vida. Revista de educación, 351, 23-47.

Santos, M. A. (2010). La formación del profesorado en las instituciones que aprenden. Revista Interuniversitaria de formación del profesorado, 24 (2), 175-200. Recuperado de: http: / / www.redalyc.org / articulo.oa?id=27419198009.

Skelton, C. (2000). A Passion for Football': Dominant Masculinities and Primary Schooling. Sport, Education and Society, 5 (1), 5-18. Doi: https: / / doi.org/10.1080/135733200114406 
Subirats, M. (2010). ¿Coeducación o escuela segregada? Un viejo y persistente debate. RASE (Revista de la Asociación de la Educación), 3, 143-158.

Subirats, M. y Tomé, A. (2010). Balones fuera (2a Ed). Barcelona: Octaedro.

Tomé González, A. (2017). Estrategias para elaborar proyectos educativos en las escuelas. Atlánticas. Revista internacional de Estudios Feministas, 2 (1), 89-116.doi: https: / / doi.org/10.17979/arief.2017.2.1.1979

Trachana, A. (2013). Espacio y poder. Ángulo recto, 5 (1), 117-131. doi: http:/ / dx.doi.org/10.5209/rev_ANRE.2013.v5.n1.42071

Vallejo, A. P. y González, J. M. (2012). Estudio sobre hábitos de actividad física saludable en niños de educación primaria de Jaén capital. Apunts. Educació Física $i$ Esports, (107), 13-23. Doi: https: / / dx.doi.org/10.5672/ apunts.2014-0983.es.(2012/1).107.01

Vega L. (2012). Conflictos y estrategias del profesorado en la aplicación de planes de igualdad en centros educativos. Tesis Doctoral. Facultad de ciencias de la educación, Universidad de Sevilla, España.

Vega L., Buzón O. y Rebollo Ma . A. (2013). Estrategias del profesorado en la aplicación de planes de Igualdad. Revista del currículum y formación del profesorado, $17,57-70$.

Villar, A., Grau, A. y Obiol, S. (2014). Educació i Gènere. Propostes per pensar $i$ repensar els gèneres. Valencia: Tirant lo Blanch.

Vizcarra, M. T., Nuño, T., Lasarte, G., Aristizabal, P. y Alvarez-Uria, A. (2015). La perspectiva de género en los títulos de Grado en la Escuela Universitaria de Magisterio de Vitoria-Gasteiz. REDU. Revista de Docencia Universitaria, 13 (1), 297-318. doi: https: / / doi.org/10.4995/redu.2015.6448

Woods, P. (1987). La escuela por dentro. Barcelona: Ediciones Paidós.

Yuen, F. y Shaw, S. M. (2003). Play: The Reproduction and Resistance of Dominant Gender Ideologies. World Leisure Journal, 45, 2, 12-21. doi: https: / / doi.org/10.1080/04419057.2003.9674312

Zaro, M. J. (1999). La identidad de género. Revista de psicoterapia, 10 (40), 5-22. doi: https: / / doi.org/10.33898/rdp.v10i40.791 